\title{
Kummer's 24 Solutions of the Hypergeometric Differential Equation with the Aid of Fractional Calculus
}

\author{
Tohru Morita ${ }^{1}$, Ken-ichi Sato ${ }^{2}$ \\ ${ }^{1}$ Tohoku University, Sendai, Japan \\ ${ }^{2}$ College of Engineering, Nihon University, Koriyama, Japan \\ Email: senmm@jcom.home.ne.jp, kensatokurume@ybb.ne.jp
}

Received 30 November 2015; accepted 24 February 2016; published 29 February 2016

Copyright (C) 2016 by authors and Scientific Research Publishing Inc.

This work is licensed under the Creative Commons Attribution International License (CC BY). http://creativecommons.org/licenses/by/4.0/

c) (i) Open Access

\begin{abstract}
We know that the hypergeometric function, which is a solution of the hypergeometric differential equation, is expressed in terms of the Riemann-Liouville fractional derivative (fD). The solution of the differential equation obtained by the Euler method takes the form of an integral, which is confirmed to be expressed in terms of the Riemann-Liouville fD of a function. We can rewrite this derivation such that we obtain the solution in the form of the Riemann-Liouville fD of a function. We present a derivation of Kummer's 24 solutions of the hypergeometric differential equation by this method.
\end{abstract}

\section{Keywords}

Fractional Derivative, Hypergeometric Differential Equation, Hypergeometric Function

\section{Introduction}

The hypergeometric function is a solution of the hypergeometric differential equation, and is known to be expressed in terms of the Riemann-Liouville fractional derivative (fD) ([1], p. 334). By the Euler method ([2], Section 3.2), the solution of the hypergeometric differential equation is obtained in the form of an integral, which is confirmed to be expressed in terms of the Riemann-Liouville $\mathrm{fD}$ of a function. This shows that we can obtain the solution in the form of the Riemann-Liouville fD of a function. In fact, Nishimoto [3] obtained a solution of the hypergeometric differential equation in terms of the Liouville $\mathrm{fD}$ in the first step, and then expressed the obtained fD in terms of the hypergeometric function in the second step. His calculation in the second step is unacceptable. In [4], he gave a derivation of Kummer's 24 solutions of the hypergeometric differential equation 
([5], Formula 15.5.4) ([6], Section 2.2) by his method. In the present paper, we show that the desired solutions are obtained by using the Riemann-Liouville fD in place of the Liouville fD.

In a preceding paper [7], we discussed the Riemann-Liouville $\mathrm{fD}$ and the Liouville $\mathrm{fD}$ as analytic continuations of the respective fractional integrals (fIs), on the basis of the papers by Lavoie et al [1] [8], and those by Nishimoto [3] and Campos [9], respectively. In Section 2, we define these fIs of a function $f(\zeta),{ }_{R} D_{\xi}^{-\lambda} f(z)$ and ${ }_{L} D_{\phi}^{-\lambda} f(z)$, of order $\lambda \in{ }_{+} \mathbb{C}$, by (1) and (2), respectively, and give their properties which we use later. The notation $\mathbb{C}$ is defined at the end of this section.

In Section 3, following [1] [3] [7]-[9], the Riemann-Liouville fD, ${ }_{C} D_{\xi}^{v} f(z)$ and ${ }_{p} D_{\xi}^{v} f(z)$, and the Liouville fD, ${ }_{H} D_{\phi}^{v} f(z)$ and ${ }_{M} D_{\phi}^{v} f(z)$, of order $v \in \mathbb{C}$, are defined in the form of a contour integral, for a function $f(\zeta)$ which is analytic on a neighborhood of the path of integration. They are defined such that they are analytic continuations of the corresponding fI as a function of $v \in \mathbb{C}$. In the present paper, the $\mathrm{fI}$ and $\mathrm{fD}$ are operated to a function of the form $\zeta^{\gamma}\left(\zeta-\xi_{2}\right)^{\gamma_{2}}$ for $\gamma \in \mathbb{C}$ and $\gamma_{2} \in \mathbb{C}$. The analytic continuations of ${ }_{R} D_{\xi}^{v} f(z)$ and ${ }_{L} D_{\phi}^{v} f(z)$ are then shown to be analytic as a function of $v$ as well as of $\gamma$ and $\gamma_{2}$. In the present paper, we use this fact in the calculation. In the following, we use fD to represent $\mathrm{fI}$ and $\mathrm{fD}$ as a whole.

In [1], the expression of the hypergeometric function: ${ }_{2} F_{1}(a, b ; c ; z)$ in terms of the Riemann-Liouville fD is given. In Sections 4 and 4.1, its derivation is presented with the aid of the method using the Riemann-Liouville fD. In Sections 4.2-4.4 and 5, Kummer's 24 solutions of the hypergeometric differential equation are derived in two ways in the present method.

In a separate paper [10], a method of obtaining the asymptotic expansion of the Riemann-Liouville fD is presented by using a relation of its expression via a path integral or a contour integral with the corresponding Liouville fD. It is then applied to obtain the asymptotic expansion of the confluent hypergeometric function which is a solution of Kummer's differential equation. In that paper, Kummer's 8 solutions of Kummer's differential equation are obtained by using the method which is adopted in the present paper to obtain the solutions of the hypergeometric differential equation.

We use notations $\mathbb{C}, \mathbb{R}$ and $\mathbb{Z}$, which represent the sets of all complex numbers, of all real numbers and of all integers, respectively. We use also the notations given by ${ }_{+} \mathbb{C}:=\{z \in \mathbb{C} \mid \operatorname{Re} z>0\}, \mathbb{Z}_{>a}:=\{n \in \mathbb{Z} \mid n>a\}$, $\mathbb{Z}_{<b}:=\{n \in \mathbb{Z} \mid n<b\}, \mathbb{Z}_{[a, b]}:=\{n \in \mathbb{Z} \mid a \leq n \leq b\}$ for $a \in \mathbb{Z}$ and $b \in \mathbb{Z}_{>a-1}$, and $\mathbb{R}_{>0}:=\{x \in \mathbb{R} \mid x>0\}$.

\section{Riemann-Liouville fD and Liouville fD}

Following preceding papers [7] [10], we adopt the following definitions of the Riemann-Liouville fI, $\phi$-dept Liouville fI and the corresponding fDs.

\subsection{Riemann-Liouville fI on the Complex Plane}

Let $\xi \in \mathbb{C}$ and $z \in \mathbb{C}$. We denote the path of integration from $\xi$ to $z$ by $P(\xi, z)$, and use $f(\zeta) \in \mathcal{L}^{1}(P(\xi, z))$ to denote that the function $f(\zeta)$ is integrable on $P(\xi, z)$.

Definition 1. Let $\xi \in \mathbb{C}, \quad z \in \mathbb{C}, f(\zeta) \in \mathcal{L}^{1}(P(\xi, z))$ and $f(\zeta)$ be continuous on a neighborhood of $\zeta=z$. Then the Riemann-Liouville fI of order $\lambda \in{ }_{+} \mathbb{C}$ is defined by

$$
{ }_{R} D_{\xi}^{-\lambda} f(z)=\frac{1}{\Gamma(\lambda)} \int_{\xi}^{z}(z-\zeta)^{\lambda-1} f(\zeta) \mathrm{d} \zeta=\frac{1}{\Gamma(\lambda)} \int_{0}^{z-\xi} \eta^{\lambda-1} f(z-\eta) \mathrm{d} \eta,
$$

where $\Gamma(\lambda)$ is the gamma function.

\subsection{Definition of $\phi$-Dept Liouville fI}

Let $z \in \mathbb{C}$ and $\phi \in \mathbb{R}$. We denote the half line $\left\{z+t \mathrm{e}^{i \phi} \mid 0<t<\infty\right\}$, by $P_{\phi}(z)$, or by $\left(z, z+\infty \cdot \mathrm{e}^{i \phi}\right)$. When $f\left(z+t \mathrm{e}^{i \phi}\right)$ is locally integrable as a function of $t$ in the interval $(0, \infty)$, we denote this by $f(\zeta) \in \mathcal{L}_{\text {loc }}^{1}\left(P_{\phi}(z)\right)$.

Definition 2. Let $z \in \mathbb{C}, \phi \in \mathbb{R}, s \in \mathbb{R}$, and $f(\zeta) \in \mathcal{L}_{\text {loc }}^{1}\left(P_{\phi}(z)\right)$. Let $s_{1}$ be such that the integral $\int_{1}^{\infty} t^{-s-1}\left|f\left(z+t \mathrm{e}^{\mathrm{i} \phi}\right)\right| \mathrm{d} t$ converges for $s>s_{1}$ and diverges for $s<s_{1}$. We then call $s_{1}$ the abscissa of convergence, and denote it by $s_{1}[f]$ or $s_{1}[f(\zeta)]$.

We then have $s_{1} \in \mathbb{R}$ or $s_{1}=-\infty$.

Lemma 1. Let $f_{\gamma, \gamma_{2}}(\zeta)=\zeta^{\gamma}\left(\zeta-\xi_{2}\right)^{\gamma_{2}}$ for $\gamma \in \mathbb{C}$ and $\gamma_{2} \in \mathbb{C}$. Then $s_{1}\left[f_{\gamma, \gamma_{2}}\right]=\operatorname{Re}\left(\gamma+\gamma_{2}\right)$. 
Definition 3. Let $z \in \mathbb{C}$ and $\phi \in \mathbb{R}$. Let $f(\zeta) \in \mathcal{L}_{\text {loc }}^{1}\left(P_{\phi}(z)\right)$ and $f(\zeta)$ be continuous on a neighborhood of $\zeta=z$. Let $\lambda \in{ }_{+} \mathbb{C}, s_{1}[f]<0$ and $\operatorname{Re} \lambda<-s_{1}[f]$. Then we define ${ }_{L} D_{\phi}^{-\lambda} f(z)$ by

$$
{ }_{L} D_{\phi}^{-\lambda} f(z)=-\frac{1}{\Gamma(\lambda)} \int_{P_{\phi}(z)}(z-\zeta)^{\lambda-1} f(\zeta) \mathrm{d} \zeta=\frac{\mathrm{e}^{i(\phi+\pi) \lambda}}{\Gamma(\lambda)} \int_{0}^{\infty} t^{\lambda-1} f\left(z-t \mathrm{e}^{\mathrm{i}(\phi+\pi)}\right) \mathrm{d} t .
$$

We call ${ }_{L} D_{\phi}^{-\lambda} f(z)$ the $\phi$-dept Liouville fI of $f(z)$.

Definition 4. When the conditions in Definition 3 are satisfied, we define ${ }_{R} D_{\xi}^{-\lambda} f(z)$ for $\xi=z+\infty \cdot \mathrm{e}^{i \phi}$ by (1).

The following lemma was mentioned in [11].

Lemma 2. Let $\xi=z+\infty \cdot \mathrm{e}^{i \phi}$. Then ${ }_{R} D_{\xi}^{-\lambda} f(z)={ }_{L} D_{\phi}^{-\lambda} f(z)$.

Proof. This is confirmed by comparing the second members of (1) and of (2).

\subsection{Definitions of Riemann-Liouville fD and Liouville fD}

Definition 5. The Riemann-Liouville $f D:{ }_{R} D_{\xi}^{v} f(z)$ for $\xi \in \mathbb{C}$ and the Liouville $f D:{ }_{L} D_{\phi}^{v} f(z)={ }_{R} D_{\xi}^{v} f(z)$ for $\xi=z+\infty \cdot \mathrm{e}^{i \phi}$, of order $v \in \mathbb{C}$ satisfying $\operatorname{Re} v \geq 0$, are defined by

$$
{ }_{R} D_{\xi}^{v} f(z)=D^{m}\left[{ }_{R} D_{\xi}^{v-m} f(z)\right],
$$

when the righthand side exists, where $m=\lfloor\operatorname{Re} v\rfloor+1$, and $D^{m} f(z)=f^{(m)}(z)$ for $m \in \mathbb{Z}_{>-1}$.

Here $\lfloor x\rfloor$ for $x \in \mathbb{R}$ denotes the greatest integer not exceeding $x$.

\subsection{Index Law and Leibniz's Rule of Riemann-Liouville fI and Liouville fI}

We use the following index law and Leibniz's rule, in Section 4.2. By Lemma 2, the formulas for $\xi=\infty \cdot \mathrm{e}^{i \phi}$ are for the Liouville fI.

Lemma 3. Let $\lambda \in{ }_{+} \mathbb{C}, v \in \mathbb{C}$ satisfy $\operatorname{Re} v \leq \operatorname{Re} \lambda$, and ${ }_{R} D_{\xi}^{-\lambda} f(z)$ exist. Then

$$
{ }_{R} D_{\xi}^{v}\left[{ }_{R} D_{\xi}^{-\lambda} f(z)\right]={ }_{R} D_{\xi}^{v-\lambda} f(z), \quad{ }_{R} D_{\xi}^{\lambda}\left[{ }_{R} D_{\xi}^{-\lambda} f(z)\right]=f(z) .
$$

Proof. Proof for $v \in \mathbb{R}$ and $\lambda \in \mathbb{R}$ is found in ([12], Section 2.2.6), where $p$ and $q$ appear in place of $-\lambda$ and $v$, respectively. The proofs there apply for $p \in \mathbb{C}$ and $q \in \mathbb{C}$ if we replace $p$ and $q$ in the inequalities there by $\operatorname{Re} p$ and $\operatorname{Re} q$, respectively.

Lemma 4. Let $\lambda \in{ }_{+} \mathbb{C}, v \in \mathbb{C}$ and $s_{1}[f]$ satisfy $s_{1}[f]<0$, and (i) $\operatorname{Re} v<0$ and $-\operatorname{Re} v+\operatorname{Re} \lambda<-s_{1}[f]$, or (ii) $0 \leq \operatorname{Re} v \leq \operatorname{Re} \lambda$ and $\operatorname{Re} \lambda+1<-s_{1}[f]$. Then (4) holds valid for $\xi=z+\infty \cdot \mathrm{e}^{\mathrm{i} \phi}$.

Proof. Proof of (4) for the case (i) is found in ([7], Appendix A). In the case (ii), with the aid of this knowledge and formula (3), we prove the first equation in (4) in the following way:

$$
D^{n}\left[{ }_{L} D_{\phi}^{\nu-n}\left[{ }_{L} D_{\phi}^{-\lambda} f(z)\right]\right]=D^{n}\left[{ }_{L} D_{\phi}^{\nu-n-\lambda} f(z)\right]=D^{n}\left[D^{-n+\delta}\left[{ }_{L} D_{\phi}^{\nu-\lambda-\delta} f(z)\right]\right]=D^{\delta}\left[{ }_{L} D_{\phi}^{\nu-\lambda-\delta} f(z)\right],
$$

where $n=\lfloor\operatorname{Re} v \mid+1, \delta=0$ if $\operatorname{Re} v<\operatorname{Re} \lambda$, and $\delta=1$ if $\operatorname{Re} v=\operatorname{Re} \lambda$. When $v=\lambda$, (5) shows the second equation in (4).

Lemma 5. Let $\lambda \in{ }_{+} \mathbb{C}$, and ${ }_{R} D_{\xi}^{-\lambda} f(z)$ exist. Then ${ }_{R} D_{\xi}^{-\lambda}[z \cdot f(z)]=z \cdot{ }_{R} D_{\xi}^{-\lambda} f(z)-\lambda \cdot{ }_{R} D_{\xi}^{-\lambda-1} f(z)$.

Proof. By using the righthand side of (1), we see that both sides of the equation in this lemma are equal to

$$
\frac{1}{\Gamma(\lambda)} \int_{0}^{z-\xi} \eta^{\lambda-1}(z-\eta) f(z-\eta) \mathrm{d} \eta .
$$

This Leibniz's rule is given in ([13], Section 5.5). The following corollary follows from this lemma.

Corollary 1. Let $\lambda \in{ }_{+} \mathbb{C}$, and ${ }_{R} D_{\xi}^{-\lambda} f(z)$ exist. Then

$$
\begin{aligned}
& z \cdot{ }_{R} D_{\xi}^{-\lambda} f(z)={ }_{R} D_{\xi}^{-\lambda}[z \cdot f(z)]+\lambda \cdot{ }_{R} D_{\xi}^{-\lambda-1} f(z), \\
& z^{2} \cdot{ }_{R} D_{\xi}^{-\lambda} f(z)={ }_{R} D_{\xi}^{-\lambda}\left[z^{2} \cdot f(z)\right]+2 \lambda \cdot{ }_{R} D_{\xi}^{-\lambda-1}[z \cdot f(z)]+\lambda(\lambda+1) \cdot{ }_{R} D_{\xi}^{-\lambda-2} f(z) .
\end{aligned}
$$




\section{Analytic Continuations of Riemann-Liouville fD and Liouville fD}

\subsection{Analytic Continuations of Riemann-Liouville fI}

In [1] [7] [8], analytic continuations of the Riemann-Liouville fI via contour integrals are discussed. In [7], ${ }_{C} D_{\xi}^{v} f(z)$ and ${ }_{P} D_{\xi}^{v} f(z)$ for $\xi \in \mathbb{C}$ are defined as follows.

Definition 6. Let $f(\zeta)$ be analytic on a neighborhood of the path $P(\xi, z)$ and on the point $Z$, and $f(\zeta) \in \mathcal{L}^{1}(P(\xi, z))$. Then ${ }_{C} D_{\xi}^{v} f(z)$ is defined by

$$
{ }_{C} D_{\xi}^{v} f(z)=\frac{\Gamma(v+1)}{2 \pi i} \int_{\xi}^{\left(z^{+}\right)}(\zeta-z)^{-v-1} f(\zeta) \mathrm{d} \zeta,
$$

for $v \in \mathbb{C} \backslash \mathbb{Z}_{<0}$, where the contour of integration is the Cauchy contour $C\left(\xi, z^{+}\right)$shown in Figure 1 (a), which starts from $\xi$, encircles the point z counterclockwise, and goes back to $\xi$, without crossing the path $P(\xi, z)$. When $-n \in \mathbb{Z}_{<0}$, we put ${ }_{C} D_{\xi}^{-n} f(z):=\lim _{v \rightarrow-n} D_{\xi}^{v} f(z)$.

Definition 7. Let $f_{\gamma}(\zeta)=(\zeta-\xi)^{\gamma} \cdot f_{0}(\zeta), \gamma \in \mathbb{C} \backslash \mathbb{Z}, f_{0}(\xi) \neq 0$, and $f_{0}(\zeta)$ be analytic on a neighborhood of the path $P(\xi, z)$ and on the points $\xi$ and z. Then ${ }_{P} D_{\xi}^{v} f_{\gamma}(z)$ is defined by

$$
{ }_{P} D_{\xi}^{v} f_{\gamma}(z)=\mathrm{e}^{-i \gamma \pi} \frac{\Gamma(v+1)}{4 \pi \sin \gamma \pi} \int_{C_{P}(\xi, z)}(\zeta-z)^{-v-1} f_{\gamma}(\zeta) \mathrm{d} \zeta,
$$

for $v \in \mathbb{C} \backslash \mathbb{Z}_{<0}$, where $C_{P}(\xi, z)$ is the Pochhammer contour shown in Figure $\mathbf{1}(\mathbf{b})$. When $-n \in \mathbb{Z}_{<0}$, we put ${ }_{P} D_{\xi}^{-n} f_{\gamma}(z):=\lim _{v \rightarrow-n P} D_{\xi}^{v} f_{\gamma}(z)$. When $\gamma=n \in \mathbb{Z}_{>-1}$, we put ${ }_{P} D_{\xi}^{v} f_{n}(z):=\lim _{\gamma \rightarrow n} D_{\xi}^{v} f_{\gamma}(z)$.

\subsection{Analytic Continuations of Liouville fI}

In [3] [7] [9], the analytic continuation of Liouville fI: ${ }_{H} D_{\phi}^{v} f(z)$ is discussed. It is defined in [7] as follows.

Definition 8. Let $f(\zeta)$ be analytic on a neighborhood of the path $P_{\phi}(z)$, and $s_{1}[f]<\infty$ and $\operatorname{Re} v>s_{1}[f]$. Then ${ }_{H} D_{\phi}^{v} f(z)$ for $v \in \mathbb{C} \backslash \mathbb{Z}_{<0}$ is defined by

$$
{ }_{H} D_{\phi}^{v} f(z)=\frac{\Gamma(v+1)}{2 \pi i} \int_{\xi}^{\left(z^{+}\right)}(\zeta-z)^{-v-1} f(\zeta) \mathrm{d} \zeta,
$$

where $\xi=z+\infty \cdot \mathrm{e}^{i \phi}$. When $-n \in \mathbb{Z}_{<0}$, we put ${ }_{H} D_{\phi}^{-n} f(z):=\lim _{v \rightarrow-n H} D_{\phi}^{v} f(z)$.

In [7], another analytic continuation of Liouville fI: ${ }_{M} D_{\phi}^{v} f(z)$ was introduced. Here we define it for a function of the form $f_{\gamma, \gamma_{2}}(\zeta)=\zeta^{\gamma}\left(\zeta-\xi_{2}\right)^{\gamma_{2}} \cdot f_{0}(\zeta)$, where $\xi_{2} \in \mathbb{C} \backslash P_{\phi}(z), \quad \gamma \in \mathbb{C} \backslash \mathbb{Z}, \quad \gamma_{2} \in \mathbb{C} \backslash \mathbb{Z}$, and $f_{0}(\zeta)$ is an entire function.

Definition 9. Let (i): $f_{\gamma, \gamma_{2}}(\zeta)$ be a function of the form stated above, where $\xi_{2} \neq 0$, (ii): $\tilde{C}$ be the modified Pochhammer contour shown in Figure 2, where $\delta \in \mathbb{R}_{>0}, \delta_{1} \in \mathbb{R}_{>0}, \quad X \in \mathbb{R}_{>0}, Y \in \mathbb{R}_{>0}, m:=\min \left(\delta, \delta_{1}\right)$ and $\quad M:=\max (X, Y)$ satisfy $m<M$, and (iii): $\phi \in \mathbb{R}, \quad z \in \mathbb{C}$ and $\xi_{2}$ satisfy $m<|z|<M$, $m<\left|z-\xi_{2}\right|<M, \quad \arg z-\phi-\pi \neq \equiv 0(\bmod 2 \pi)$, and $\arg (z-\xi)-\phi-\pi \neq \equiv(\bmod 2 \pi)$, Then ${ }_{M} D_{\phi}^{v} f_{\gamma, \gamma_{2}}(z)$ for $v \in \mathbb{C} \backslash \mathbb{Z}_{<0}$ is defined by

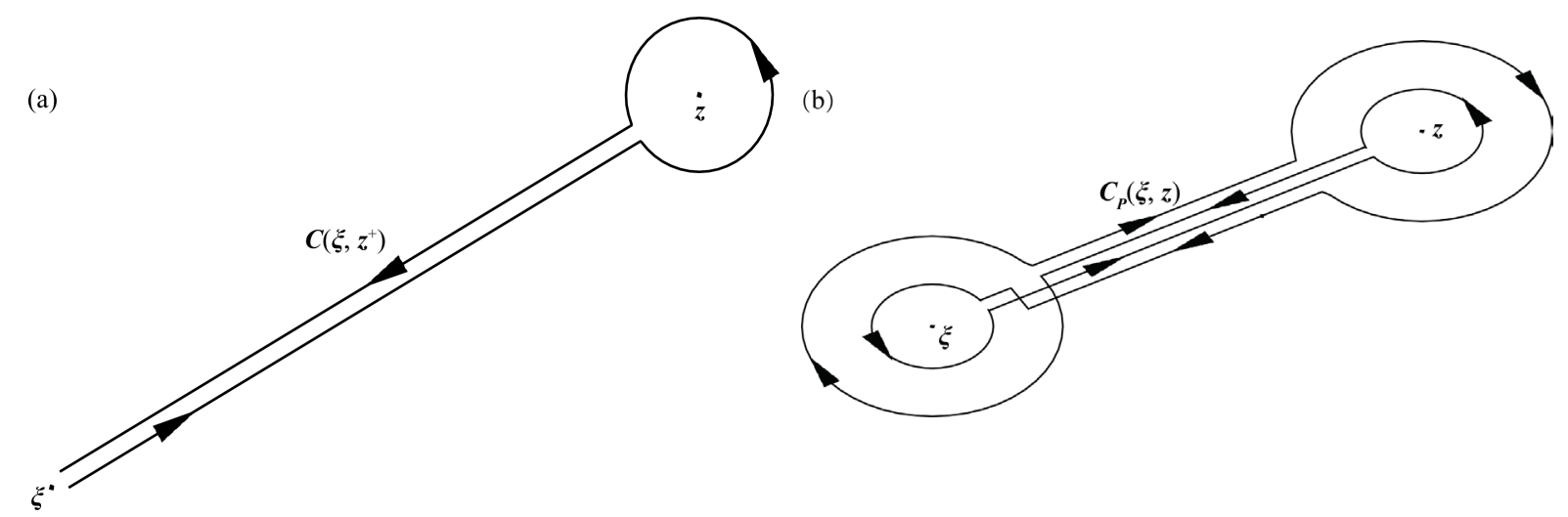

Figure 1. The contours of integration, (a): $C\left(\xi, z^{+}\right),(b): C_{P}(\xi, z)$. 


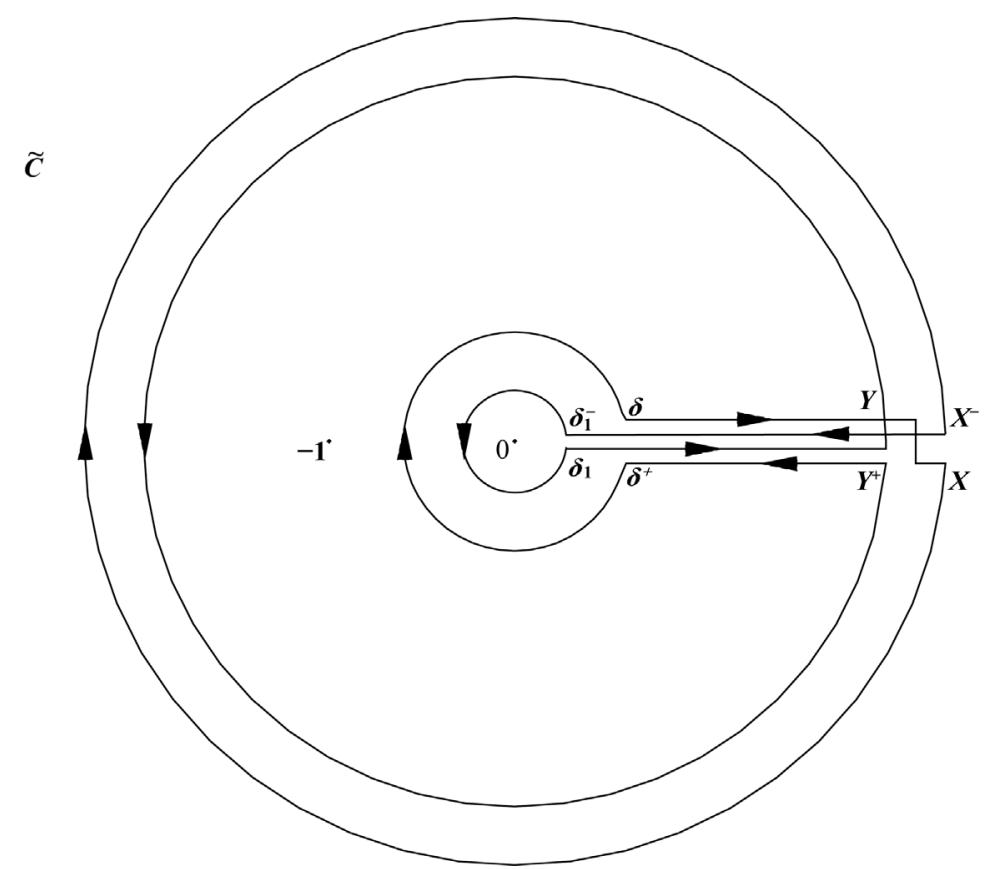

Figure 2. The contour of integration $\tilde{C}$, from $\delta$, to $X$, to $X^{-}=X \mathrm{e}^{-2 i \pi}$, $\delta_{1}^{-}=\delta_{1} \mathrm{e}^{-2 i \pi}, \delta_{1}, Y, Y^{+}=Y \mathrm{e}^{2 i \pi}, \delta^{+}=\delta \mathrm{e}^{2 i \pi}$, and then back to $\delta$.

$$
{ }_{M} D_{\phi}^{v} f_{\gamma, \gamma_{2}}(z)=\mathrm{e}^{-i \phi v+i \hat{\gamma} \pi} \frac{\Gamma(v+1)}{4 \pi \sin \hat{\gamma} \pi} \int_{\tilde{C}} \eta^{-v-1} f_{\gamma, \gamma_{2}}\left(z+\eta \mathrm{e}^{i \phi}\right) \mathrm{d} \eta,
$$

where $\hat{\gamma}:=\gamma+\gamma_{2}-v \notin \mathbb{Z}$. When $-n \in \mathbb{Z}_{<0}$, we put ${ }_{M} D_{\phi}^{-n} f_{\gamma, \gamma_{2}}(z):=\lim _{v \rightarrow-n}{ }_{M} D_{\phi}^{v} f_{\gamma, \gamma_{2}}(z)$. When $\gamma+\gamma_{2}-v \in \mathbb{Z}$, ${ }_{M} D_{\phi}^{v} f_{\gamma, \gamma_{2}}(z)$ is defined by analyticity.

\subsection{Analyticity of Riemann-Liouville fD and Liouville fD}

In this section, we consider functions $f_{1}(\zeta)$ and $f_{2}(\zeta)$ expressed by

$$
f_{1}(\zeta)=(\zeta-\xi)^{\gamma}\left(\zeta-\xi_{2}\right)^{\gamma_{2}}, \quad f_{2}(\zeta)=\zeta^{\gamma}\left(\zeta-\xi_{2}\right)^{\gamma_{2}},
$$

where $\xi \in \mathbb{C}, \xi_{2} \in \mathbb{C}, \gamma \in \mathbb{C} \backslash \mathbb{Z}$ and $\gamma_{2} \in \mathbb{C} \backslash \mathbb{Z}$.

The following Lemmas $6 \sim 10$ are obtained by modifying the corresponding arguments given in Section 2 for the Riemann-Liouville fD and in Sections 3.1 3.3 for the Liouville fD in [7], with the aid of ([14], Sections 3.1 and 3.2).

Lemma 6. ${ }_{C} D_{\xi}^{v} f_{1}(z)$ and ${ }_{H} D_{\phi}^{v} f_{2}(z)$ are analytic as a function of $v \in \mathbb{C}$ as well as of $\gamma_{2} \in \mathbb{C}$, and of $\gamma \in \mathbb{C}$ in the domains $\operatorname{Re} \gamma>-1$ and $\operatorname{Re} \gamma+\gamma_{2}>-1$, respectively.

Lemma 7. ${ }_{P} D_{\xi}^{v} f_{1}(z)$ and ${ }_{M} D_{\phi}^{v} f_{2}(z)$ are analytic as a function of $v \in \mathbb{C}$ as well as of $\gamma \in \mathbb{C}$ and $\gamma_{2} \in \mathbb{C}$.

Lemma 8. Let ${ }_{C} D_{\xi}^{v} f_{1}(z)$ exist. Then ${ }_{R} D_{\xi}^{v} f_{1}(z)$ exists and ${ }_{C} D_{\xi}^{v} f_{1}(z)={ }_{R} D_{\xi}^{v} f_{1}(z)$.

Lemma 9. Let ${ }_{P} D_{\xi}^{v} f_{1}(z)$ exist. If $\gamma+1 \in{ }_{+} \mathbb{C}$, then ${ }_{C} D_{\xi}^{v} f_{1}(z)$ exists and ${ }_{P} D_{\xi}^{v} f_{1}(z)={ }_{C} D_{\xi}^{v} f_{1}(z)$.

Lemma 10. Lemmas 8 and 9 with ${ }_{R} D_{\xi}^{v} f_{1}(z),{ }_{C} D_{\xi}^{v} f_{1}(z),{ }_{P} D_{\xi}^{v} f_{1}(z)$ and $\gamma$, replaced by ${ }_{L} D_{\phi}^{v} f_{2}(z)$, ${ }_{H} D_{\phi}^{v} f_{2}(z),{ }_{M} D_{\phi}^{v} f_{2}(z)$ and $\gamma+\gamma_{2}$, respectively, are valid.

Remark 1. The statements related with ${ }_{H} D_{\phi}^{v} f_{2}(z)$ and ${ }_{M} D_{\phi}^{v} f_{2}(z)$ in Lemma 10 are proved by modifying the proofs of Theorems 3.1 and 3.3, respectively, in [7].

In the following sections, we use ${ }_{C} D_{\xi}^{v} f(z)$ and ${ }_{P} D_{\xi}^{v} f_{\gamma}(z)$ for the Riemann-Liouville fD.

\section{The Hypergeometric Function in Terms of Riemann-Liouville fD}

Let $a \in \mathbb{C}, b \in \mathbb{C}, c \in \mathbb{C}$ and $z \in \mathbb{C}$ satisfy (i): $c \notin \mathbb{Z}_{\triangleleft 1}$ or (ii): $c=-m \in \mathbb{Z}_{<1}$ and either $-m<a=-n \in \mathbb{Z}_{<1}$ 
or $-m<b=-n \in \mathbb{Z}_{\triangleleft 1}$. In the case (i), the hypergeometric series ${ }_{2} F_{1}(a, b ; c ; z)$ is defined by

$$
{ }_{2} F_{1}(a, b ; c ; z)=\sum_{k=0}^{\infty} \frac{(a)_{k}(b)_{k}}{(c)_{k} k !} z^{k}, \quad|z|<1,
$$

where $(z)_{k}=\prod_{l=0}^{k-1}(z+l)$ for $k \in \mathbb{Z}_{>0}$ and $(z)_{0}=1$, for $z \in \mathbb{C}$. In the case (ii), it is defined by

$$
{ }_{2} F_{1}(a, b ;-m ; z)=\sum_{k=0}^{n} \frac{(a)_{k}(b)_{k}}{(-m)_{k} k !} z^{k} .
$$

The integral representation of ${ }_{2} F_{1}(a, b ; c ; z)$ is given by

$$
{ }_{2} F_{1}(a, b ; c ; z)=\frac{\Gamma(c)}{\Gamma(c-a) \Gamma(a)} \int_{0}^{1} t^{a-1}(1-t)^{c-a-1}(1-t z)^{-b} \mathrm{~d} t,
$$

when $\operatorname{Re} c>\operatorname{Re} a>0$, in ([5], Formula 15.5.4) ([6], Section 2.5). In fact, we obtain (13) from (14) by expanding the righthand side of the latter in powers of $z$ and then performing the integration term-by-term, when $|z|<1$.

This function is a solution of the hypergeometric differential equation:

$$
z(1-z) \frac{\mathrm{d}^{2} w}{\mathrm{~d} z^{2}}+[c-(1+a+b) z] \frac{\mathrm{d} w}{\mathrm{~d} z}-a b \cdot w=0,
$$

which has also another solution given by

$$
z^{1-c} \cdot{ }_{2} F_{1}(1+a-c, 1+b-c ; 2-c ; z),
$$

see ([5], Section 15.5.1) ([6], Section 2.2).

\subsection{Solution of the Hypergeometric Differential Equation (15) with the Aid of Riemann-Liouville fD}

The function ${ }_{2} F_{1}(a, b ; c ; z)$ is known to be expressed in the form of (18) for $l=3$ given below, in [1]. We now obtain the solutions of (15) expressed in terms of the Riemann-Liouville fD.

Proofs of the following two lemmas are presented in the following two sections.

Lemma 11. Let $p_{l}(z)$ and $w_{l}(\xi, z)$ for $l \in \mathbb{Z}_{[1,4]}$ be as follows:

$$
\begin{aligned}
& p_{1}(z)=1, \quad p_{2}(z)=(1-z)^{c-a-b}, \quad p_{3}(z)=z^{1-c}, \quad p_{4}(z)=z^{1-c}(1-z)^{c-a-b}, \\
& w_{l}(\xi, z)=p_{l}(z) \gamma_{l}(\xi){ }_{P} D_{\xi}^{a_{l}-1}\left[z^{a_{l}-c_{l}}(1-z)^{c_{l}-b_{l}-1}\right],
\end{aligned}
$$

where the values $a_{l}, b_{l}$ and $c_{l}$ are given in Table 1 , and $\gamma_{l}(\xi)$ are constants. Then $w_{l}(\xi, z)$, for $l \in \mathbb{Z}_{[1,4]}$ and $\xi \in\left\{0,1, \infty \cdot \mathrm{e}^{\mathrm{i} \phi}\right\}$, are solutions of (15).

Lemma 12. When $\xi=0$, we choose $\gamma_{l}(0)=\frac{\Gamma\left(2-c_{l}\right)}{\Gamma\left(1+a_{l}-c_{l}\right)}$, and then $w_{l}(\xi, z)$ given by (18) are expressed as

$$
w_{l}(0, z)=p_{l}(z) z^{1-c_{l}} \cdot{ }_{2} F_{1}\left(1+a_{l}-c_{l}, 1+b_{l}-c_{l} ; 2-c_{l} ; z\right), \quad l \in \mathbb{Z}_{[1,4]} .
$$

\begin{tabular}{cccc} 
Table 1. Values of $a_{l}, b_{l}$ and $c_{l}$. & & \\
\hline$l$ & $a_{l}$ & $b_{l}$ & $c_{l}$ \\
\hline 1 & $a$ & $b$ & $c$ \\
2 & $c-a$ & $c-b$ & $c$ \\
3 & $1-c+a$ & $1-c+b$ & $2-c$ \\
4 & $1-a$ & $1-b$ & $2-c$
\end{tabular}


Corollary 2. When we put $w_{l}(z)=w_{l}(0, z)$ for $l \in \mathbb{Z}_{[1,4]}$, we have

$$
\begin{aligned}
& w_{1}(z)=z^{1-c} \cdot{ }_{2} F_{1}(1+a-c, 1+b-c ; 2-c ; z), \\
& w_{2}(z)=(1-z)^{c-a-b} z^{1-c} \cdot{ }_{2} F_{1}(1-a, 1-b ; 2-c ; z), \\
& w_{3}(z)={ }_{2} F_{1}(a, b ; c ; z), \\
& w_{4}(z)=(1-z)^{c-a-b} \cdot{ }_{2} F_{1}(c-a, c-b ; c ; z) .
\end{aligned}
$$

Remark 2. The solutions $w_{l}(z)$ given in Corollary 2 satisfy $w_{1}(z)=w_{2}(z)$ and $w_{3}(z)=w_{4}(z)$; see ([5], Formulas 15.5.3 15.5.4) ([6], Section 2.2). This is confirmed by noting that the solution of (15) in the form $\mathrm{z}^{\lambda} \sum_{k=0}^{\infty} c_{k} z^{k}$ with a fixed $\lambda$ and $c_{0}=1$ is unique.

\subsection{Proof of Lemma 11}

Lemma 13 Let $\operatorname{Re} a<-1$, and (i): $\xi=0$ and $\operatorname{Re}(a-c)>-1$, or (ii): $\xi=1$ and $\operatorname{Re}(c-b)>0$, or (iii): $\xi=\infty \cdot \mathrm{e}^{i \phi}, \operatorname{Re}(1+a-b)<0$ and $\operatorname{Re} b>1$. Then a solution of (15) is given by

$$
w(z)={ }_{R} D_{\xi}^{a-1}\left[z^{a-c}(1-z)^{c-b-1}\right] .
$$

Proof. We assume that a solution of (15) is expressed as $w(z)={ }_{R} D_{\xi}^{-\lambda} v(z)$ for $\lambda \in \mathbb{C}$ satisfying $\operatorname{Re} \lambda>2$. If (i) or (ii) applies, we substitute this $w(z)$ in (15), and use Lemma 3 and Corollary 1. We then obtain

$$
\begin{aligned}
& { }_{R} D_{\xi}^{2-\lambda}[z(1-z) \cdot v(z)]+{ }_{R} D_{\xi}^{1-\lambda}[((3-2 \lambda-a-b) z-2+\lambda+c) \cdot v(z)] \\
& -(1-\lambda-a)(1-\lambda-b) \cdot{ }_{R} D_{\xi}^{-\lambda} v(z)=0 .
\end{aligned}
$$

Putting $\lambda=1-a$ and hence assuming $\operatorname{Re} a<-1$, and applying ${ }_{R} D_{\xi}^{\lambda-2}$ to (23), we obtain

$$
z(1-z) \cdot v(z)+D^{-1}[((1+a-b) z-1-a+c) \cdot v(z)]=0,
$$

with the aid of Lemma 3. This equation requires that

$$
\frac{\mathrm{d}}{\mathrm{d} z}[z(1-z) \cdot v(z)]+((1+a-b) z-1-a+c) \cdot v(z)=0,
$$

and $z(1-z) \cdot v(z)=0$ when $z=\xi$. Now we obtain $v(z)=z^{a-c}(1-z)^{c-b-1}$ if any of the three conditions in Lemma 13 is satisfied. Thus we obtain (22). When (iii) applies, we use Lemma 4 in place of Lemma 3. Then we have to use $s_{1}[z(1-z) v(z)]=a-b+1$.

Remark 3. The proof of Lemma 13 corresponds to the derivation, given in ([2], pp. 43-44), of an integral form of the solution of (15), where the method is called the Euler method.

Lemma 14. If $w(a, b, c ; z)$ is a solution of (15), then $p_{l}(z) w\left(a_{l}, b_{l}, c_{l} ; z\right)$ for $l \in \mathbb{Z}_{[2,4]}$ also are solutions of (15).

Proof. We first consider the case of $l=3$. We replace $w(z)$ by $z^{\lambda} \cdot u(z)$ in (15), then we obtain

$$
z^{2}(1-z) u^{\prime \prime}+[(2 \lambda+1+a+b) z+2 \lambda+c] z \cdot u^{\prime}-(\lambda+a)(\lambda+b) z \cdot u+(\lambda-1+c) \lambda \cdot u=0 .
$$

When we choose $\lambda=1-c$, this equation is reduced to (15) with $a, b, c$ and $w$ replaced by $a_{3}, b_{3}, c_{3}$ and $u$, respectively. In the case of $l=2$, we use $(1-z)^{\lambda} u(z)$ in place of $z^{\lambda} \cdot u(z)$. By using this lemma for $l=3$ and $l=2$, we see that $p_{3}(z) w\left(a_{3}, b_{3}, c_{3} ; z\right)$ and $p_{3}(z) p_{2}(z) w\left(c_{3}-a_{3}, c_{3}-b_{3}, c_{3} ; z\right)$ are solutions of (15). This proves the case of $l=4$.

Proof of Lemma 11. The formula (18) for $w_{l}(\xi, z)$ follows from Lemmas 13 and 14 with the aid of Lemmas 7-10.

\subsection{Expressions of $w_{l}(\xi, z)$ in Terms of the Hypergeometric Functions}

We now present the expressions of $w_{l}(\xi, z)$ given in (18) in terms of the hypergeometric functions. We then obtain Kummer's 24 solutions. In the following section, we give another derivation of them.

Proof of Lemma 12 is given at the first part of the proof of Lemma 15 below. 
By using Lemmas 8, 9 and 10 and the middle member of (1), (18) is expressed as

$$
w_{l}(\xi, z)=p_{l}(z) \frac{\gamma_{l}(\xi)}{\Gamma\left(1-a_{l}\right)} \int_{\xi}^{z} \eta^{a_{l}-c_{l}}(z-\eta)^{-a_{l}}(1-\eta)^{c_{l}-b_{l}-1} \mathrm{~d} \eta
$$

Lemma 15. We choose $\gamma_{l}(0)=\frac{\Gamma\left(2-c_{l}\right)}{\Gamma\left(1+a_{l}-c_{l}\right)}, \quad \gamma_{l}(1)=\frac{\Gamma\left(1-a_{l}-b_{l}+c_{l}\right)}{\Gamma\left(c_{l}-b_{l}\right)}$, and $\gamma_{l}(\infty)=\frac{\Gamma\left(1-a_{l}+b_{l}\right)}{\Gamma\left(b_{l}\right)}$. Then $w_{l}(\xi, z)$ given by (18) is expressed as $w_{l}(\xi, z)=\tilde{w}_{l}(\xi, z)(-1)^{d_{k, l}}$, where

$$
\begin{aligned}
\tilde{w}_{l}(\xi, z) & =p_{l}(z) \frac{\gamma_{l}(\xi)}{\Gamma\left(1-a_{l}\right)} z^{\alpha_{k, l}}(1-z)^{\beta_{k, l}} \int_{0}^{1} t^{A_{k, l}-1}(1-t)^{C_{k, l}-A_{k, l}-1}\left(1-t \cdot \zeta_{k}\right)^{-B_{k, l}} \mathrm{~d} t \\
& =p_{l}(z) z^{\alpha_{k, l}}(1-z)^{\beta_{k, l}} \cdot{ }_{2} F_{1}\left(A_{k, l}, B_{k, l} ; C_{k, l} ; \zeta_{k}\right), \quad l \in \mathbb{Z}_{[1,4]},
\end{aligned}
$$

if $k, \zeta_{k}, \xi, d_{k, l}, \alpha_{k, l}, \beta_{k, l}, A_{k, l}, B_{k, l}, C_{k, l}$ are those given in a row in Table 2.

Proof. We put $\xi=0$ and $\eta=z t$ in (27). Then we obtain

$$
w_{l}(0, z)=p_{l}(z) \frac{\gamma_{l}(0)}{\Gamma\left(1-a_{l}\right)} z^{1-c_{l}} \int_{0}^{1} t^{a_{l}-c_{l}}(1-t)^{-a_{l}}(1-z t)^{c_{l}-b_{l}-1} \mathrm{~d} t,
$$

when $1-\operatorname{Re} a_{l}>0$, and also $1+\operatorname{Re}\left(a_{l}-c_{l}\right)>0$. The data in the row $k=1$ in Table 2, are so chosen that $w(\xi, z)$ given by (28) with the data is equal to (30). Lemma 12 follows from (30) with the aid of formula (19).

We put $\xi=1$ and $\eta=1-t(1-z)$ in (27). We then obtain

$$
w_{l}(1, z)=p_{l}(z) \frac{\gamma_{l}(1)}{\Gamma\left(1-a_{l}\right)}(-1)^{1-a_{l}}(1-z)^{c_{l}-a_{l}-b_{l}} \int_{0}^{1} t^{c_{l}-b_{l}-1}(1-t)^{-a_{l}}(1-t(1-z))^{a_{l}-c_{l}} \mathrm{~d} t,
$$

when $\operatorname{Re}\left(c_{l}-b_{l}\right)>0, \operatorname{Re} a_{l}<1$ and $|1-z|<1$. The data in the row $k=2$ are taken from this equation.

We put $\xi=\infty$ and $\eta=\frac{z}{t}$ in (27). We then obtain

$$
w_{l}(\infty, z)=p_{l}(z) \frac{\gamma_{l}(\infty)}{\Gamma\left(1-a_{l}\right)}(-1)^{c_{l}-a_{l}-b_{l}} z^{-b_{l}} \int_{0}^{1} t^{b_{l}-1}(1-t)^{-a_{l}}\left(1-\frac{t}{z}\right)^{c_{l}-b_{l}-1} \mathrm{~d} t,
$$

when $\operatorname{Re} b_{l}>0$, Re $a_{l}<1$ and $|z|>1$. The data in the row $k=3$ are taken from this equation.

We put $t=1-x$ in (28). Then we obtain

Table 2. Functions $\zeta_{k}$ of $z$ and values of $\xi, d_{k, l}, \quad \alpha_{k, l}, \quad \beta_{k, l}, A_{k, l}, \quad B_{k, l}$ and $C_{k, l}$, for $k \in \mathbb{Z}_{[1,6]}$.

\begin{tabular}{ccccccccc}
\hline$k$ & $\zeta_{k}$ & $\xi$ & $d_{k, l}$ & $\alpha_{k, l}$ & $\beta_{k, l}$ & $A_{k, l}$ & $B_{k, l}$ & $C_{k, l}$ \\
\hline 1 & $z$ & 0 & 0 & $1-c_{l}$ & 0 & $1+a_{l}-c_{l}$ & $1+b_{l}-c_{l}$ & $2-c_{l}$ \\
2 & $1-z$ & 1 & $1-a_{l}$ & $a_{l}-c_{l}$ & $c_{l}-a_{l}-b_{l}$ & $c_{l}-b_{l}$ & $c_{l}-a_{l}$ & $1+c_{l}-a_{l}-b_{l}$ \\
3 & $\frac{1}{z}$ & $\infty$ & $c_{l}-a_{l}-b_{l}$ & $-b_{l}$ & 0 & $b_{l}$ & $1+b_{l}-c_{l}$ & $1-a_{l}+b_{l}$ \\
4 & $1-\frac{1}{z}$ & 1 & $1-a_{l}$ & $a_{l}-c_{l}$ & $c_{l}-a_{l}-b_{l}$ & $1-a_{l}$ & $c_{l}-a_{l}$ & $1+c_{l}-a_{l}-b_{l}$ \\
5 & $\frac{1}{1-z}$ & $\infty$ & $1-a_{l}$ & $1-c_{l}$ & $c_{l}-b_{l}-1$ & $1-a_{l}$ & $1+b_{l}-c_{l}$ & $1-a_{l}+b_{l}$ \\
6 & $1-\frac{1}{1-z}$ & 0 & 0 & $1-c_{l}$ & $c_{l}-b_{l}-1$ & $1-a_{l}$ & $1+b_{l}-c_{l}$ & $2-c_{l}$ \\
\hline
\end{tabular}




$$
\begin{aligned}
w_{l}(\xi, z)= & p_{l}(z) \frac{\gamma_{l}(\xi)}{\Gamma\left(1-a_{l}\right)}(-1)^{d_{k, l}} z^{\alpha_{k, l}}(1-z)^{\beta_{k, l}}\left(1-\zeta_{k}\right)^{-B_{k, l}} \\
& \times \int_{0}^{1} x^{C_{k, l}-A_{k, l}-1}(1-x)^{A_{k, l}-1}\left(1-x \cdot \frac{\zeta_{k}}{\zeta_{k}-1}\right)^{-B_{k, l}} \mathrm{~d} x .
\end{aligned}
$$

Applying this to the formula (28) for $k=1,2$ and 3, we obtain the results in Table 2 for $k=6,4$ and 5, respectively.

Remark 4. Let $\tilde{w}_{l}(\xi, z)$ given by (29), for $k \in \mathbb{Z}_{[1,6]}$ and $l \in \mathbb{Z}_{[1,4]}$, be denoted by $\tilde{w}_{n}(z)$ when $n=4(k-1)+l$. We show that they give Kummer's 24 solutions of $(15)$, which are $w_{n}(z)$ for $\mathbb{Z}_{[1,24]}$ given in Theorem 1 below. They are related by $\tilde{w}_{n}(z)=w_{n}(z)(-1)^{d_{n}}$ for $n \in \mathbb{Z}_{[1,12]}$, and by $\tilde{w}_{n}(z)=w_{m(n)}(z)(-1)^{\tilde{d}_{n}}$ for $n \in \mathbb{Z}_{[13,24]}$, where $m(13)=m(15)=14, m(14)=m(16)=15, m(17)=18, m(18)=20, m(19)=17$, $m(20)=19, m(21)=22, m(22)=21, m(23)=24, m(24)=23$, and $\tilde{d}_{n} \in \mathbb{R}$ for $n \in \mathbb{Z}_{[1,24]}$. Here $w_{14}(z)$ and $w_{15}(z)$ appear twice, and $w_{13}(z)$ and $w_{16}(z)$ do not appear. We note that when the formers are solutions of (15), the latters which are obtained from the formers by exchanging a and $b$, are obviously solutions of (15). By adding these in the set of solutions $\tilde{w}_{n}(z)$, we have the 24 solutions of (15).

Remark 5. In Lemma 15, we have two expressions of $w_{l}(\xi, z)$ for different $k$. For instance for $\xi=0$ and $l=3$, we have $\tilde{w}_{3}(z)=w_{3}(0, z)=\tilde{w}_{23}(z)$, which is given in ([5], Formulas 15.3.3 15.3.5) ([6], Section 2.4.1).

Remark 6. When $\xi=\infty$, we have ${ }_{R} D_{\infty}^{-\lambda} f(z)={ }_{L} D_{0}^{-\lambda} f(z)$, so that the equation (32) and the data for $k=3$ in Table 2 are obtained by using the Liouville fD, and is given by Nishimoto in [4]. In that case, Nishimoto's derivation is justified.

\subsection{Solutions of (15) as a Function of $1-z, \frac{1}{z}, 1-\frac{1}{z}, \frac{1}{1-z}$, and $1-\frac{1}{1-z}$}

In the following, there appear $\tilde{p}_{k}(z), \quad \zeta_{k}, \tilde{b}_{k}$ and $\tilde{c}_{k}$ for $k \in \mathbb{Z}_{[2,6]}$. They are listed in Table 3.

Lemma 16. If $w(a, b, c ; z)$ is a solution of $(15)$, then $\tilde{p}_{k}(z) w\left(a, \tilde{b}_{k}, \tilde{c}_{k} ; \zeta_{k}\right)$ for $k \in \mathbb{Z}_{[2,6]}$ also are solutions of (15).

Proof. When $k=2$, we replace $z$ and $w(z)$ by $1-\zeta_{2}$ and $y_{2}\left(\zeta_{2}\right)$, respectively, in (15). We then obtain the same equation with $c, z$ and $w(z)$ replaced by $\tilde{c}_{2}, \zeta_{2}$ and $y_{2}\left(\zeta_{2}\right)$, respectively. We call the obtained Equation (15-2).

When $k=3$, we put $\zeta=\zeta_{3}$, and replace $z$ and $w(z)$ by $\frac{1}{\zeta}$ and $z^{-\lambda} y(\zeta)$ or $\zeta^{\lambda} y(\zeta)$, respectively, in (15). We then obtain

$$
\zeta^{2}(\zeta-1) y^{\prime \prime}+[(2 \lambda+2-c) \zeta+(-2 \lambda-1+a+b)] \zeta \cdot y^{\prime}+[(\lambda+1-c) \lambda \cdot \zeta-(\lambda-a)(\lambda-b)] y=0
$$

When we choose $\lambda=a$, this equation is reduced to (15) with $b, c, z$ and $w(z)$ replaced by $\tilde{b}_{3}, \tilde{c}_{3}, \zeta$ and $y(\zeta)$, respectively. We call the obtained Equation (15-3).

Table 3. Fuctions $\tilde{p}_{k}(z)$ and $\zeta_{k}$ of $z$, and values of $\tilde{b}_{k}$ and $\tilde{c}_{k}$, for $k \in \mathbb{Z}_{[2,6]}$.

\begin{tabular}{ccccc}
\hline$k$ & $\tilde{p}_{k}(z)$ & $\zeta_{k}$ & $\tilde{b}_{k}$ & $\tilde{c}_{k}$ \\
\hline 2 & 1 & $1-z$ & $b$ & $1+a+b-c$ \\
3 & $z^{-a}$ & $\frac{1}{z}$ & $1+a-c$ & $1+a-b$ \\
4 & $z^{-a}$ & $1-\frac{1}{z}$ & $1+a-c$ & $1+a-b$ \\
5 & $(1-z)^{-a}$ & $\frac{1}{1-z}$ & $c-b$ & $c$ \\
\hline
\end{tabular}


When $k=4$, we replace $\zeta$ and $y(\zeta)$ by $1-\zeta_{4}$ and $y_{4}\left(\zeta_{4}\right)$, respectively, in (15-3). We then obtain the same equation with $\tilde{b}_{3}, \tilde{c}_{3}, \zeta$ and $y(\zeta)$ replaced by $\tilde{b}_{4}=\tilde{b}_{3}, \tilde{c}_{4}=1+a+\tilde{b}_{3}-\tilde{c}_{3}, \zeta_{4}$ and $y_{4}\left(\zeta_{4}\right)$, respectively.

When $k=5$, we replace $\zeta_{2}$ and $y_{2}\left(\zeta_{2}\right)$ by $\frac{1}{\zeta_{5}}$ and $\zeta_{2}^{-a} y_{5}\left(\zeta_{5}\right)$ or $\zeta_{5}^{a} y\left(\zeta_{5}\right)$, respectively, in (15-2).

We then obtain the same equation with $b, c_{2}, \zeta_{2}$ and $y_{2}\left(\zeta_{2}\right)$ replaced by $\tilde{b}_{5}=1+a-\tilde{c}_{2}, \tilde{c}_{5}=1+a-b$, $\zeta_{5}$ and $y_{5}\left(\zeta_{5}\right)$, respectively. We call the obtained Equation (15-5).

When $k=6$, we replace $\zeta_{5}$ and $y_{5}\left(\zeta_{5}\right)$ by $1-\zeta_{6}$ and $y_{6}\left(\zeta_{6}\right)$, respectively, in (15-5). We then obtain the same equation with $b_{5}, c_{5}, \zeta_{5}$ and $y_{5}\left(\zeta_{5}\right)$ replaced by $\tilde{b}_{6}=\tilde{b}_{5}, \tilde{c}_{6}=1+a+\tilde{b}_{5}-\tilde{c}_{5}, \zeta_{6}$ and $y_{6}\left(\zeta_{6}\right)$, respectively.

By Corollary 2 and Lemma 16, we obtain the following corollary.

Corollary 3. Let $w_{l}(a, b, c ; z)$ for $l \in \mathbb{Z}_{[1,4]}$ represent the righthand side of the equation for $w_{l}(z)$ given in (20) (21). Then for $n=4(k-1)+l, \quad l \in \mathbb{Z}_{[1,4]}$ and $k \in \mathbb{Z}_{[2,6]}$,

$$
w_{n}(z)=\tilde{p}_{k}(z) w_{l}\left(a, \tilde{b}_{k}, \tilde{c}_{k} ; \zeta_{k}\right)
$$

is a solution of (15).

We note here the following remark, which is used in obtaining Table 4 below.

Table 4. Functions $z_{n}$ of $z$ and values of $k, l, \alpha_{n}, \beta_{n}, A_{n}, B_{n}$ and $C_{n}$ for $n \in \mathbb{Z}_{[1,24]}$.

\begin{tabular}{ccccccccc}
\hline$n$ & $z_{n}$ & $k$ & $l$ & $\alpha_{n}$ & $\beta_{n}$ & $A_{n}$ & $B_{n}$ & $C_{n}$ \\
\hline 1 & $z$ & 1 & 1 & $1-c$ & 0 & $1+a-c$ & $1+b-c$ & $2-c$ \\
2 & $z$ & 1 & 2 & $1-c$ & $c-a-b$ & $1-a$ & $1-b$ & $2-c$ \\
3 & $z$ & 1 & 3 & 0 & 0 & $a$ & $b$ & $c$ \\
4 & $z$ & 1 & 4 & 0 & $c-a-b$ & $c-a$ & $c-b$ & $c$ \\
5 & $1-z$ & 2 & 1 & $c-a-b$ & 0 & $c-b$ & $c-a$ & $1-a-b+c$ \\
6 & $1-z$ & 2 & 2 & $c-a-b$ & $1-c$ & $1-a$ & $1-b$ & $1-a-b+c$ \\
7 & $1-z$ & 2 & 3 & 0 & 0 & $a$ & $b$ & $1+a+b-c$ \\
8 & $1-z$ & 2 & 4 & 0 & $1-c$ & $1+b-c$ & $1+a-c$ & $1+a+b-c$ \\
9 & $z^{-1}$ & 3 & 1 & $b$ & 0 & $b$ & $1+b-c$ & $1-a+b$ \\
10 & $z^{-1}$ & 3 & 2 & $b$ & $c-a-b$ & $1-a$ & $c-a$ & $1-a+b$ \\
11 & $z^{-1}$ & 3 & 3 & $a$ & 0 & $a$ & $1+a-c$ & $1+a-b$ \\
12 & $z^{-1}$ & 3 & 4 & $a$ & $c-a-b$ & $1-b$ & $c-b$ & $1+a-b$ \\
13 & $1-z^{-1}$ & 4 & 1 & $c-a-b$ & $a$ & $c-b$ & $1-b$ & $1-a-b+c$ \\
14 & $1-z^{-1}$ & 4 & 2 & $c-a-b$ & $b$ & $1-a$ & $c-a$ & $1-a-b+c$ \\
15 & $1-z^{-1}$ & 4 & 3 & 0 & $a$ & $a$ & $1+a-c$ & $1+a+b-c$ \\
16 & $1-z^{-1}$ & 4 & 4 & 0 & $b$ & $1+b-c$ & $b$ & $1+a+b-c$ \\
17 & $(1-z)^{-1}$ & 5 & 1 & $b$ & 0 & $b$ & $c-a$ & $1-a+b$ \\
18 & $(1-z)^{-1}$ & 5 & 2 & $b$ & $1-c$ & $1-a$ & $1+b-c$ & $1-a+b$ \\
19 & $(1-z)^{-1}$ & 5 & 3 & $a$ & 0 & $a$ & $c-b$ & $1+a-b$ \\
20 & $(1-z)^{-1}$ & 5 & 4 & $a$ & $1-c$ & $1-b$ & $1+a-c$ & $1+a-b$ \\
21 & $z(z-1)^{-1}$ & 6 & 1 & $1-c$ & $a$ & $1+a-c$ & $1-b$ & $2-c$ \\
22 & $z(z-1)^{-1}$ & 6 & 2 & $1-c$ & $b$ & $1-a$ & $1+b-c$ & $2-c$ \\
23 & $z(z-1)^{-1}$ & 6 & 3 & 0 & $a$ & $a$ & $c-b$ & $c$ \\
24 & $z(z-1)^{-1}$ & 6 & 4 & 0 & $b$ & $c-a$ & $b$ & $c$ \\
\hline & & & & & & & & \\
\hline
\end{tabular}


Remark $7 \quad \tilde{p}_{2}(z)=1, \quad \tilde{p}_{k}(z)=\zeta_{k}^{a}$ for $k=3$ and 5, and $\tilde{p}_{k}(z)=\left(1-\zeta_{k}\right)^{a}$ for $k=4$ and 6.

\section{Kummer's 24 Solutions of the Hypergeometric Differential Equation}

By Corollary 3 and Lemma 7, we obtain the following theorem by the present method.

Theorem 1 We have 24 solutions of (15), which are expressed as

$$
w_{n}(z)=z_{n}^{\alpha_{n}}\left(1-z_{n}\right)^{\beta_{n}}{ }_{2} F_{1}\left(A_{n}, B_{n} ; C_{n} ; z_{n}\right), \quad n \in \mathbb{Z}_{[1,24]},
$$

where the functions $z_{n}$ of $z$ and the values of $\alpha_{n}, \beta_{n}, A_{n}, B_{n}$ and $C_{n}$ are listed in Table 4.

The values for $n \in \mathbb{Z}_{[1,4]}$ in Table 4 are obtained by comparing (35) with (20) (21) in Corollary 2. By Corollary 3 and Remark 7, the functions $z_{n}$ and the values for $n \in \mathbb{Z}_{[5,24]}$ are obtained with the aid of the following lemma.

Lemma 17. Let $\tilde{\alpha}_{l}(a, b, c), \quad \tilde{\beta}_{l}(a, b, c), \quad \tilde{A}_{l}(a, b, c), \quad \tilde{B}_{l}(a, b, c)$ and $\tilde{C}_{l}(a, b, c)$ for $l \in \mathbb{Z}_{[1,4]}$ represent $\alpha_{l}, \beta_{l}, A_{l}, B_{l}$ and $C_{l}$, respectively, as a function of $a, b$ and $c$. Then the values of $\alpha_{n}, \beta_{n}, A_{n}, B_{n}$ and $C_{n}$ and functions $z_{n}$ of $z$ for $n \in \mathbb{Z}_{[5,24]}$ are given by

$$
\begin{aligned}
& \alpha_{n}=\tilde{\alpha}_{l}\left(a, \tilde{b}_{k}, \tilde{c}_{k}\right), \quad \beta_{n}=\tilde{\beta}_{l}\left(a, \tilde{b}_{k}, \tilde{c}_{k}\right), \quad n \in \mathbb{Z}_{[5,8]}, \\
& \alpha_{n}=a+\tilde{\alpha}_{l}\left(a, \tilde{b}_{k}, \tilde{c}_{k}\right), \quad \beta_{n}=\tilde{\beta}_{l}\left(a, \tilde{b}_{,}, \tilde{c}_{k}\right), \quad n \in \mathbb{Z}_{[9,12]} \cup \mathbb{Z}_{[17,20]}, \\
& \alpha_{n}=\tilde{\alpha}_{l}\left(a, \tilde{b}_{k}, \tilde{c}_{k}\right), \quad \beta_{n}=a+\tilde{\beta}_{l}\left(a, \tilde{b}_{k}, \tilde{c}_{k}\right), \quad n \in \mathbb{Z}_{[13,16]} \cup \mathbb{Z}_{[21,24]}, \\
& A_{n}=\tilde{A}_{l}\left(a, \tilde{b}_{k}, \tilde{c}_{k}\right), \quad B_{n}=\tilde{B}_{l}\left(a, \tilde{b}_{k}, \tilde{c}_{k}\right), \quad C_{n}=\tilde{C}_{l}\left(a, \tilde{b}_{k}, \tilde{c}_{k}\right), \quad Z_{n}=\zeta_{k}, \quad n \in \mathbb{Z}_{[5,24]},
\end{aligned}
$$

where $k=\lfloor n / 4\rfloor+1$ and $l=n-4(k-1)$.

The following lemma is well known, see ([5], Formulas 15.5.3 15.5.14) ([6], Section 2.2).

Theorem 2. The solutions $w_{n}(z)$ given in Theorem 1 for $n \in \mathbb{Z}_{[1,24]}$ are related by

$$
w_{2 n-1}(z)=w_{2 n}(z), \quad n \in \mathbb{Z}_{[1,12]} \text {. }
$$

Proof. This is confirmed by using Lemma 16 or Corollary 3 with the aid of Remark 2.

\section{References}

[1] Lavoie, J.L., Tremblay, R. and Osler, T.J. (1975) Fundamental Properties of Fractional Derivatives via Pochhammer Integrals. In: Ross, B., Ed., Fractional Calculus and its Applications, Springer-Verlag, Berlin, 327-356. http://dx.doi.org/10.1007/BFb0067118

[2] Sneddon, I.N. (1980) Special Functions of Mathematical Physics and Chemistry. Longman Inc., New York.

[3] Nishimoto, K. (1991) An Essence of Nishimoto’s Fractional Calculus. Descartes Press, Koriyama.

[4] Nishimoto, K. (1997) Kummer's Twenty-Four Functions and N-Fractional Calculus. Nonlinear Analysis, Theory, Methods \& Applications, 30, 1271-1282.

[5] Abramowitz, M. and Stegun, I.A. (1972) Handbook of Mathematical Functions with Formulas, Graphs and Mathematical Tables, Chapter 15. Dover Publ., Inc., New York.

[6] Magnus, W., Oberhettinger, F. and Soni, R.P. (1966) Formulas and Theorems for the Special Fuctions of Mathematical Physics, Chapter II. Springer-Verlag New York Inc., New York. http://dx.doi.org/10.1007/978-3-662-11761-3

[7] Morita, T. and Sato, K. (2013) Liouville and Riemann-Liouville Fractional Derivatives via Contour Integrals. Fractional Calculus and Applied Analysis, 16, 630-653. http://dx.doi.org/10.2478/s13540-013-0040-9

[8] Lavoie, J.L., Osler, T.J. and Tremblay, R. (1976) Fractional Derivatives and Special Functions. SIAM Review, 18, 240268. http://dx.doi.org/10.1137/1018042

[9] Campos, L.M.B.C. (1984) On a Concept of Derivative of Complex Order with Applications to Special Functions. IMA Journal of Applied Mathematics, 33, 109-133. http://dx.doi.org/10.1093/imamat/33.2.109

[10] Morita, T. and Sato, K. (2015) Asymptotic Expansions of Fractional Derivatives and Their Applications. Mathematics 3, 171-189. http://dx.doi.org/10.3390/math3020171

[11] Samko, S.G., Kilbas, A.A. and Marichev, O.I. (1993) Fractional Integrals and Derivatives, Theory and Applications. 
Gordon and Breach Sc. Publ., Amsterdam.

[12] Podlubny, I. (1999) Fractional Differential Equations. Academic Press, San Diego.

[13] Oldham, K.B. and Spanier, J. (2006) The Fractional Calculus. Dover Publ. Inc., New York.

[14] Whittaker, E.T. and Watson, G.N. (1935) A Course of Modern Analysis. Cambridge U.P., Cambridge. 